\title{
Ideal Gas Thermodynamic Functions of the Isotopic Hydrogen Sulfides
}

\author{
Lester Haar, ${ }^{2}$ Joe C. Bradley, and Abraham S. Friedman
}

\begin{abstract}
The ideal gas thermodynamic functions for $\mathrm{H}_{2} \mathrm{~S}, \mathrm{D}_{2} \mathrm{~S}, \mathrm{~T}_{2} \mathrm{~S}, \mathrm{HDS}$, HTS, and D'TS were calculated from molecular data. The recent spectroscopic data for $\mathrm{H}_{2} \mathrm{~S}$ and $\mathrm{D}_{2} \mathrm{~S}$ by Allen $[1]^{3}$ were employed. The zero-order frequencies for the other isotopes were obtained by a normal coordinate treatment. The partition functions are obtained in closed form. The calculations include high-temperature corrections for vibrational anharmonicity, rotationvibration coupling, centrifugal stretching, and a low-temperature correction for nonclassical rotation. The statistical calculations were performed on the Bureau's digital computer. Tables of $C_{p}^{\circ} / R,\left(H^{\circ}-E_{0}^{\circ}\right) / R T,-\left(F^{\circ}-E_{0}^{\circ}\right) / R T$, and $S^{\circ} / R$ have been calculated at close temperature intervals from $50^{\circ}$ to $5,000^{\circ} \mathrm{K}$.
\end{abstract}

\section{Introduction}

Procedures were developed in earlier papers $[2,3]$ for the National Bureau of Standards Eastern Automatic Computer-SEAC, to calculate the ideal gas thermodynamic functions for a variety of molecules. A closed form analytical expression for the partition function is used, which includes terms for anharmonicity, rotation-vibration interaction, centrifugal stretching, and nonclassical rotation. The internal partition function used in the calculations is

$$
\begin{array}{r}
Q=\frac{1}{S}\left[\Pi_{i}\left(1-e^{\left.-u_{i}\right)^{-1}}\right]\left[\frac{\pi}{\sigma_{A} \sigma_{B} \sigma_{C}}\right]^{1 / 2}\left[1+\frac{\theta_{1}}{T}+\frac{\theta_{2}}{T^{2}}\right] \times\right. \\
\\
{[1+\rho T]\left[\prod_{i \leq j}\left(1+f_{i j}\right)\right]\left[\Pi_{i}\left(1+1 / 2 \frac{\delta_{i}}{\left(e^{\left.u_{i}-1\right)}\right)}\right)\right] .}
\end{array}
$$

The rotational partition function for an asymmetric top was derived by Stripp and Kirkwood [4] and Woolley [5].

This expression is

$$
Q_{\mathrm{rot}}=\left[\frac{\pi}{\sigma_{A} \sigma_{B} \sigma_{C}}\right]^{1 / 2} f\left(\sigma_{A}, \sigma_{B}, \sigma_{C}\right) \text { where } \sigma=\frac{h^{2}}{8 \pi^{2} k T I}
$$

The function $f\left(\sigma_{A}, \sigma_{B}, \sigma_{C}\right)$ has the form

$$
f\left(\sigma_{A}, \sigma_{B}, \sigma_{C}\right)=1+\frac{\theta_{1}}{T}+\frac{\theta_{2}}{T^{2}}+\cdots \cdots
$$

where the $\theta_{i}$ are constants. Woolley [5] also calculated ortho-para effects, not included in this expression.

The other internal contributions considered in the partition function are

1 This work was supported in part by the U. S. Atomic Energy Commission, Division of Research.

2 Present address: Johns Hopkins University Applied Physics Laboratory.

Present address. Johns Hopkins University Applied Physics Laboratory.

$$
\begin{aligned}
& Q_{\text {centrifugal stretching }}=1+\rho T \\
& Q_{\text {anharmonicity }}=\Pi_{i \leq j}\left(1+f_{i j}\right), \\
& Q_{\substack{\text { rotation-vibration } \\
\text { coupling }}}=I_{i}\left(1+1 / 2 \frac{\delta_{i}}{\left(e^{u_{i}}-1\right)}\right),
\end{aligned}
$$

and $S$ is the symmetry number. In these expressions $\rho$ represents the centrifugal stretching constant, and $\delta_{i}$ are the rotation-vibration coupling constants;

$$
f_{i j}=\frac{X_{i j} \frac{h c}{k T}}{\left(e^{u_{i}}-1\right)\left(e^{u_{j}}-1\right)}
$$

where the $X_{i j}$ are the observed vibrational anharmonicity coefficients, and

$$
u_{i}=\nu_{i} \frac{h c}{k T}
$$

where $\nu_{i}$ are the observed fundamentals.

Details of the method of computation are found in [2].

The thermodynamic functions can be expressed in terms of $\ln ?$ or the derivatives of $Q$.

$$
\begin{aligned}
\frac{F^{\circ}-E_{0}^{\circ}}{R T} & =-\ln Q \\
\frac{H^{\circ}-E_{0}^{\circ}}{R T} & =-\frac{Q^{\prime}}{Q T} \\
\frac{S^{\circ}}{R} & =\frac{Q^{\prime}}{Q T}+\ln Q \\
\frac{C^{\circ}}{R} & =\frac{Q^{\prime \prime}}{Q T^{2}}-\frac{Q^{\prime 2}}{Q T}
\end{aligned}
$$

where $Q^{\prime}=\frac{d Q}{d\left(\frac{1}{T}\right)}$ and $Q^{\prime \prime}=\frac{d^{2} Q}{d\left(\frac{1}{T}\right)^{2}}$. 


\section{Molecular Constants}

The molecular constants used are based primarily on data for $\mathrm{H}_{2} \mathrm{~S}$ and $\mathrm{D}_{2} \mathrm{~S}$, reported by Allen [1]. A normal coordinate analysis yielded zero order vibrational frequencies for HDS, HTS and $\mathrm{T}_{2} \mathrm{~S}$. The values for the centrifugal stretching constants calculated by Allen are $\rho_{\mathrm{H}_{2} \mathrm{~s}}=1.68 \times 10^{-5}\left({ }^{\circ} \mathrm{K}\right)^{-1}$ and $\rho_{\mathrm{D}_{2} \mathrm{~s}}=1.78 \times 10^{-5}\left({ }^{\circ} \mathrm{K}\right)^{-1}$. For the calculation of the thermodynamic functions of the six isotopic hydrogen sulfide molecules, $1.7 \times 10^{-5}\left({ }^{\circ} \mathrm{K}\right)^{-1}$ was used for this constant.

The anharmonicity and rotation-vibration interaction constants for $\mathrm{T}_{2} \mathrm{~S}$, HDS, HTS, and DTS were calculated from the relations

$$
X_{i j}^{\prime}=X_{i j}\left(\frac{\omega_{i}^{\prime} \omega_{i}^{\prime}}{\omega_{i} \omega_{j}}\right)
$$

and

$$
\delta_{i}^{\prime}=\delta_{i}\left(\frac{\omega_{i}^{\prime}}{\omega_{i}}\right)
$$

respectively, where the prime quantities refer to the reference molecule.

This procedure for estimating anharmonicity and rotation-vibration coupling constants is applicable only to molecules of the same symmetry class as the

TABLE 1. Comparison of observed and calculated anharmonic constants of $\mathrm{H}_{2} \mathrm{~S}$ and $\mathrm{D}_{2} \mathrm{~S}$

$X_{i j}$ in $\mathrm{cm}^{-1}$

\begin{tabular}{|c|c|c|c|c|}
\hline & \multicolumn{2}{|c|}{$\mathrm{H}_{2} \mathrm{~S}$} & \multicolumn{2}{c|}{$\mathrm{D}_{2} \mathrm{~S}$} \\
\cline { 2 - 3 } \cline { 5 - 5 } & Allen & Calculated & Allen & Calculated \\
\cline { 5 - 6 } & & & & \\
\cline { 5 - 5 }$X_{11}$ & 25 & 22 & 13 & 11 \\
$X_{22}$ & 5.5 & 9.1 & 3.0 & 4.7 \\
$X_{33}$ & 24 & 23 & 12 & 12 \\
$X_{12}$ & 24 & 8.0 & 12 & 4.3 \\
$X_{13}$ & 94 & 81 & 50 & 42 \\
$X_{23}$ & 19 & 10 & 10 & 5.4 \\
& & & & \\
\hline
\end{tabular}

reference molecule. $\mathrm{H}_{2} \mathrm{~S}$ was used as the reference molecule for $\mathrm{T}_{2} \mathrm{~S}$. In order to obtain estimates of these constants for the three $\mathrm{X}-\mathrm{Y}-\mathrm{Z}$ type molecules, HDS, HTS, and DTS, the reference molecule HDO was employed in the above expressions. This extension of the isotope relations was tested by comparing the experimental values for $\mathrm{H}_{2} \mathrm{~S}$ and $\mathrm{D}_{2} \mathrm{~S}$ [1] with those calculated using $\mathrm{H}_{2} \mathrm{O}$ as a reference molecule. The comparison is given in table 1.

The chemical atomic weight of sulfur used in the calculations of the translational entropies was obtained from the values listed by Wichers [6]. The isotopic masses used in the zero-point frequency and equilibrium moment of inertia calculations are the values listed by Mattauch and Fluegge [7].

The molecular constants used in the calculations are listed in table 2.

\section{Thermal Properties}

The heat capacity, enthalpy, free energy, and entropy for $\mathrm{H}_{2} \mathrm{~S}$, HDS, $\mathrm{D}_{2} \mathrm{~S}$, HTS, DTS, and $\mathrm{T}_{2} \mathrm{~S}$ are tabulated in dimensionless units in tables 3 through 8. The very small changes in the constants of table 2 , obtained by using the other isotopes of sulfur in the compound formulas, together with the very large relative abundance of $\mathrm{S}^{32}$, result in a completely negligible difference between the properties of XYS ${ }^{32}$ tabulated here and the naturally occurring mixture of $\mathrm{XYS}^{32}, \mathrm{XYS}^{33}, \mathrm{XYS}^{34}$, and $\mathrm{XYS}^{36}$. The contributions of nuclear spin and isotopic mixing have been omitted; the contribution of excited electronic states, only significant near the highest temperatures, have been omitted. The values computed by Woolley [5] of the ortho-para effect for $\mathrm{H}_{2} \mathrm{~S}$ at $50^{\circ}$ and $100^{\circ} \mathrm{K}$ are 0.024 and 0.00001 respectively for $\left(C_{p}^{\circ} / R\right)$ symmetric $-\left(C_{p}^{\circ} / R\right)$ antisymmetric. These are not included in the values tabulated in table 3 . For $\mathrm{D}_{2} \mathrm{~S}$ and $\mathrm{T}_{2} \mathrm{~S}$ the effects are appreciably smaller and have been likewise omitted from the tables. Tables 3 through 8 also include the first differences for the tabulated thermodynamic functions.

\begin{tabular}{|c|c|c|c|c|c|c|c|}
\hline & Units & $\mathrm{H}_{2} \mathrm{~S}$ & HDS & $\mathrm{D}_{2} \mathrm{~S}$ & HTS & DTS & $\mathrm{T}_{2} \mathrm{~S}$ \\
\hline $\begin{array}{l}\nu_{1} \\
\nu_{2} \\
\nu_{3}\end{array}$ & $\mathrm{~cm}^{-1} \mathrm{~cm}^{-1} \mathrm{~cm}-1$ & $\begin{array}{l}2616 \\
1183 \\
2625\end{array}$ & $\begin{array}{l}1910 \\
1039 \\
2636\end{array}$ & $\begin{array}{l}1895 \\
856.0 \\
1906\end{array}$ & $\begin{array}{l}1556 \\
983.0 \\
2636\end{array}$ & $\begin{array}{l}1594 \\
791.0 \\
1921\end{array}$ & $\begin{array}{l}1585 \\
713.8 \\
1594\end{array}$ \\
\hline $\begin{array}{l}X_{11} \\
X_{12}\end{array}$ & $\mathrm{~cm}^{-1} \ldots$ & $\frac{25}{5.5}$ & $\stackrel{21}{5.5}$ & $\begin{array}{c}13 \\
3.0\end{array}$ & $\begin{array}{c}14 \\
4.9\end{array}$ & $\begin{array}{l}6.8 \\
3.1\end{array}$ & $\begin{array}{l}7.6 \\
3.2\end{array}$ \\
\hline $\begin{array}{l}\boldsymbol{X}_{33} \\
\boldsymbol{X}_{12} \\
\boldsymbol{X}_{13} \\
\boldsymbol{X}_{23}\end{array}$ & $\begin{array}{l}\mathrm{cm}^{-1} \\
\mathrm{~cm}^{-1} \\
\mathrm{~cm}^{-1}\end{array}$ & $\begin{array}{l}24 \\
22 \\
94 \\
19\end{array}$ & $\begin{array}{l}41 \\
4.3 \\
6.3 \\
11\end{array}$ & $\begin{array}{l}12 \\
12 \\
50 \\
10\end{array}$ & $\begin{array}{r}41 \\
3.4 \\
5.2 \\
10\end{array}$ & $\begin{array}{r}21 \\
2.7 \\
3.7 \\
5.9\end{array}$ & $\begin{array}{r}8.1 \\
3.0 \\
29 \\
3.7\end{array}$ \\
\hline$\delta_{1}$ & dimensionless..... & 0.051 & 0.033 & 0.037 & 0.027 & 0.028 & 0.031 \\
\hline $\begin{array}{l}\delta_{2} \\
\delta_{3}\end{array}$ & $\begin{array}{l}\text { dimensionless ..... } \\
\text { dimensionless ..... }\end{array}$ & $\begin{array}{r}-.039 \\
.036\end{array}$ & $\begin{array}{r}-.061 \\
.044\end{array}$ & $\begin{array}{r}-.028 \\
.026\end{array}$ & $\begin{array}{r}-.058 \\
.045\end{array}$ & $\begin{array}{r}-.046 \\
.033\end{array}$ & $\begin{array}{r}-.024 \\
.022\end{array}$ \\
\hline$I_{e}^{A}$ & $\mathrm{~g} \mathrm{~cm}{ }^{2} \times 10^{-40} \ldots \ldots$ & 2. 694 & 2.863 & 5.083 & 2.967 & 5.448 & 7.210 \\
\hline$I_{e}^{B}$ & $\mathrm{~g} \mathrm{~cm}^{2} \times 10^{-40} \ldots$ & 3.103 & 5.667 & 6. 201 & 8. 133 & 8.429 & 9. 286 \\
\hline$I_{*}^{C}$ & $\mathrm{~g} \mathrm{~cm}^{2} \times 10^{-40} \ldots \ldots$ & 5.797 & 8.530 & 11. 28 & 11.10 & 13.88 & 16.50 \\
\hline $\begin{array}{l}\theta_{1} \\
\theta_{2} \\
\rho\end{array}$ & $\begin{array}{l}{ }^{\circ} \mathrm{K} \\
\left({ }^{\circ} \mathbf{K}\right)^{2} \\
\left({ }^{\circ} \mathbf{K}\right){ }^{-1} \times 10^{-5}\end{array}$ & $\begin{array}{l}\text { 2. } 4306 \\
\text { 4. } 8078 \\
1.7\end{array}$ & $\begin{array}{l}\text { 1. } 5732 \\
2.2275 \\
1.7\end{array}$ & $\begin{array}{l}\text { 1. } 1897 \\
1.5140 \\
1.7\end{array}$ & $\begin{array}{l}\text { 1. } 2089 \\
\text { 1. } 3155 \\
1.7\end{array}$ & $\begin{array}{l}0.96680 \\
.84065 \\
\text { 1. }\end{array}$ & $\begin{array}{l}0.81330 \\
.59567 \\
1.7\end{array}$ \\
\hline
\end{tabular}

TABLE 2. Molecular constants 
TABLE 3. Ideal gas thermodynamic functions for $\mathrm{H}_{2} \mathrm{~S}$

\begin{tabular}{|c|c|c|c|c|c|c|c|c|}
\hline \multirow{2}{*}{${ }^{{ }^{\circ} \mathrm{K}}$} & \multicolumn{2}{|c|}{$\frac{C_{p}^{\circ}}{R}$} & \multicolumn{2}{|c|}{$\frac{\left(H^{\circ}-E_{0}^{\circ}\right)}{R T}$} & \multicolumn{2}{|c|}{$\frac{-\left(F^{\circ}-E_{0}^{\circ}\right)}{R T}$} & $\frac{S}{I}$ & \\
\hline & 4. 00286 & 2 & 3. 95091 & 866 & 13. 59988 & 72115 & 17.55080 & 72981 \\
\hline 60 & 4. 00288 & 13 & 3. 95957 & 619 & 14. 32103 & 61086 & 18. 28061 & 61705 \\
\hline 70 & 4. 00301 & 20 & 3. 96576 & 467 & 14. 93189 & 52988 & 18.89766 & 53454 \\
\hline 80 & 4. 00321 & 24 & 3. 97043 & 366 & 15. 46177 & 46787 & 19.43220 & 47153 \\
\hline 90 & 4. 00345 & 28 & 3. 97409 & 295 & 15.92964 & 41887 & 19.90373 & 42182 \\
\hline 100 & 4. 00373 & 31 & 3.97704 & 244 & 16. 34851 & 37917 & 20. 32555 & 38161 \\
\hline 110 & 4. 00404 & 39 & 3. 97948 & 206 & 16. 72768 & 34635 & 20.70716 & 34841 \\
\hline 120 & 4. 00443 & 51 & 3. 98154 & 178 & 17. 07403 & 31876 & 21.05557 & 32055 \\
\hline 130 & 4. 00494 & 72 & 3. 98332 & 157 & 17. 39279 & 29526 & 21. 37612 & 29682 \\
\hline 140 & 4. 00566 & 103 & 3. 98489 & 141 & 17. 68805 & 27498 & 21. 67294 & 27640 \\
\hline 150 & 4. 00669 & 148 & 3. 98630 & 132 & 17. 96303 & 25731 & 21. 94934 & 25863 \\
\hline 160 & 4. 00817 & 204 & 3. 98762 & 127 & 18. 22034 & 24179 & 22. 20797 & 24305 \\
\hline 170 & 4. 01021 & 272 & 3. 98889 & 125 & 18. 46213 & 22803 & 22.45102 & 22929 \\
\hline 180 & 4. 01293 & 353 & 3. 99014 & 129 & 18. 69016 & 21577 & 22. 68031 & 21706 \\
\hline 190 & 4. 01646 & 443 & 3. 99143 & 136 & 18. 90593 & 20477 & 22.89737 & 20612 \\
\hline 200 & 4. 02089 & 540 & 3. 99279 & 146 & 19.11070 & 19484 & 23.10349 & 19631 \\
\hline 210 & 4. 02629 & 640 & 3. 99425 & 160 & 19. 30554 & 18585 & 23. 29980 & 18745 \\
\hline 220 & 4. 03269 & 743 & 3. 99585 & 176 & 19. 49139 & 17766 & 23.48725 & 17942 \\
\hline 230 & 4. 04012 & 844 & 3. 99761 & 194 & 19. 66905 & 17018 & 23. 66667 & 17212 \\
\hline 240 & 4. 04856 & 944 & 3. 99955 & 215 & 19.83923 & 16331 & 23.83879 & 16546 \\
\hline 250 & 4. 05800 & 1040 & 4. 00170 & 236 & 20.00254 & 15700 & 24. 00425 & 15936 \\
\hline 260 & 4. 06840 & 1130 & 4. 00406 & 259 & 20.15954 & 15116 & 24. 16361 & 15375 \\
\hline 270 & 4. 07970 & 1215 & 4. 00665 & 283 & 20.31070 & 14576 & 24. 31736 & 14858 \\
\hline 280 & 4. 09185 & 1293 & 4. 00948 & 306 & 20.45646 & 14075 & 24. 46594 & 14382 \\
\hline 290 & 4. 10478 & 1366 & 4. 01254 & 330 & 20.59721 & 13609 & 24. 60976 & 13938 \\
\hline 300 & 4. 11844 & 1432 & 4. 01584 & 354 & 20.73330 & 13174 & 24. 74914 & 13528 \\
\hline 310 & 4. 13276 & 1491 & 4. 01938 & 377 & 20. 86504 & 12767 & 24.88442 & 13144 \\
\hline 320 & 4. 14767 & 1546 & 4. 02315 & 401 & 20. 99271 & 12386 & 25. 01586 & 12787 \\
\hline 330 & 4. 16313 & 1595 & 4. 02716 & 423 & 21.11657 & 12028 & 25.14373 & 12452 \\
\hline 340 & 4. 17908 & 1640 & 4. 03139 & 445 & 21. 23685 & 11692 & 25. 26825 & 12137 \\
\hline 350 & 4. 19548 & 1680 & 4. 03584 & 467 & 21. 35377 & 11376 & 25. 38962 & 11843 \\
\hline 360 & 4. 21228 & 1717 & 4. 04051 & 487 & 21.46753 & 11077 & 25. 50805 & 11564 \\
\hline 370 & 4. 22945 & 1752 & 4. 04538 & 508 & 21. 57830 & 10795 & 25. 62369 & 11303 \\
\hline 380 & 4. 24697 & 1782 & 4. 05046 & 526 & 21. 68625 & 10529 & 25. 73672 & 11054 \\
\hline 390 & 4. 26479 & 1811 & 4. 05572 & 546 & 21. 79154 & 10275 & 25.84726 & 10821 \\
\hline 400 & 4. 28290 & 9429 & 4. 06118 & 2982 & 21. 89429 & 48002 & 25.95547 & 50984 \\
\hline 450 & 4. 37719 & 9955 & 4. 09100 & 3355 & 22. 37431 & 43273 & 26. 46531 & 46629 \\
\hline 500 & 4. 47674 & 10382 & 4. 12455 & 3671 & 22.80704 & 39481 & 26. 93160 & 43152 \\
\hline 550 & 4. 58056 & 10705 & 4. 16126 & 3939 & 23. 20185 & 36375 & 27. 36312 & 40313 \\
\hline 600 & 4. 68761 & 10909 & 4. 20065 & 4164 & 23.56560 & 33786 & 27. 76625 & 37951 \\
\hline 650 & 4. 79670 & 10981 & 4. 24229 & 4352 & 23. 90346 & 31597 & 28. 14576 & 35949 \\
\hline 700 & 4. 90651 & 10923 & 4. 28581 & 4503 & 24. 21943 & 29722 & 28. 50525 & 34225 \\
\hline 750 & 5. 01574 & 10751 & 4. 33084 & 4618 & 24.51665 & 28098 & 28.84750 & 32715 \\
\hline 800 & 5. 12325 & 10481 & 4. 37702 & 4699 & 24. 79763 & 26676 & 29. 17465 & 31376 \\
\hline 850 & 5. 22806 & 10139 & 4. 42401 & 4751 & 25. 06439 & 25421 & 29. 48841 & 30171 \\
\hline 900 & 5. 32945 & 9744 & 4. 47152 & 4773 & 25. 31860 & 24304 & 29. 79012 & 29078 \\
\hline 950 & 5. 42689 & 9314 & 4. 51925 & 4773 & 25.56164 & 23303 & 30.08090 & 28075 \\
\hline 1000 & 5. 52003 & 8866 & 4. 56698 & 4751 & 25. 79467 & 22397 & 30.36165 & 27149 \\
\hline 1050 & 5. 60869 & 8410 & 4. 61449 & 4712 & 26. 01864 & 21576 & 30. 63314 & 26287 \\
\hline 1100 & 5. 69279 & 7960 & 4. 66161 & 4658 & 26. 23440 & 20824 & 30.89601 & 25483 \\
\hline 1150 & 5. 77239 & 7518 & 4. 70819 & 4593 & 26. 44264 & 20136 & 31.15084 & 24728 \\
\hline 1200 & 5. 84757 & 13774 & 4. 75412 & 8951 & 26. 64400 & 38408 & 31.39812 & 47360 \\
\hline 1300 & 5. 98531 & 12224 & 4. 84363 & 8600 & 27. 02808 & 36213 & 31.87172 & 44812 \\
\hline 1400 & 6. 10755 & 10843 & 4. 92963 & 8222 & 27. 39021 & 34293 & 32. 31984 & 42515 \\
\hline 1500 & 6. 21598 & 9632 & 5. 01185 & 7832 & 27. 73314 & 32598 & 32. 74499 & 40431 \\
\hline 1600 & 6. 31230 & 8576 & 5. 09017 & 7446 & 28.05912 & 31084 & 33.14930 & 38530 \\
\hline 1700 & 6. 39806 & 7661 & 5. 16463 & 7069 & 28. 36996 & 29723 & 33. 53460 & 36792 \\
\hline 1800 & 6. 47467 & & & 6707 & & 28487 & 33.90252 & \\
\hline 1900 & 6. 54336 & 6182 & 5. 30239 & 6362 & 28. 95206 & 27361 & 34. 25446 & 33723 \\
\hline 2000 & 6. 60518 & 5588 & 5. 36601 & 6036 & 29. 22567 & 26328 & 34. 59169 & 32364 \\
\hline 2100 & 6. 66106 & 5070 & 5. 42637 & 5730 & 29.48895 & 25377 & 34. 91533 & 31106 \\
\hline 2200 & 6. 71176 & 4621 & 5. 48367 & 5441 & 29. 74272 & 24497 & 35. 22639 & 29939 \\
\hline 2300 & & 4227 & & 5172 & & 23680 & 35.52578 & \\
\hline 2400 & 6. 80024 & 3882 & 5. 58980 & 4921 & 30.22449 & 22919 & 35.81430 & 27840 \\
\hline 2500 & 6. 83906 & 3580 & 5. 63901 & 4685 & 30.45368 & 22209 & 36.09270 & 26894 \\
\hline 2600 & 6.87486 & 3312 & 5. 68586 & 4466 & 30.67577 & 21543 & 36. 36164 & 26008 \\
\hline 2700 & 6. 90798 & 3076 & 5. 73052 & 4261 & 30.89120 & 20918 & 36.62172 & 25179 \\
\hline 2800 & & & & & & & & \\
\hline 2900 & 6. 96740 & 2678 & 5. 81382 & 3891 & 31. 30368 & 19776 & 37.11751 & 23666 \\
\hline 3000 & 6. 99418 & 4872 & 5.85273 & 7289 & 31.50144 & 38009 & 37.35417 & 45298 \\
\hline 3200 & 7. 04290 & 4331 & 5. 92562 & 6702 & 31.88153 & 36128 & 37.80715 & 42830 \\
\hline 3400 & 7. 08621 & & 5. 99264 & & 32. 24281 & 34430 & 38. 23545 & 40616 \\
\hline 3600 & 7. 12508 & 3520 & 6. 05449 & 5729 & 32.58711 & 32890 & 38. 64161 & 38619 \\
\hline 3800 & 7. 16028 & 3213 & 6,11178 & 5324 & 32,91601 & 31487 & 39.02780 & 36810 \\
\hline 4000 & 7. 19241 & 2954 & 6. 16502 & 4964 & 33. 23088 & 30201 & 39.39590 & 35165 \\
\hline 4200 & 7. 22195 & 2731 & 6. 21466 & 4641 & 33. 53289 & 29018 & 39. 74755 & 33660 \\
\hline & 7. 24926 & & 6. 26107 & 4352 & 33. 82307 & 27929 & 40.08415 & 32281 \\
\hline 4600 & 7. 27464 & 2370 & 6. 30459 & 4092 & 34. 10236 & 26920 & 40.40696 & 31011 \\
\hline 4800 & 7. 29834 & 2222 & 6. 34551 & 3856 & 34.37156 & 25982 & 40.71707 & 29839 \\
\hline 5000 & 7. 32056 & .... & 6. 38407 & .... & 34.63138 & -.... & 41.01546 & -.... \\
\hline
\end{tabular}

TABLE 4. Ideal gas thermodynamic functions for HDS

\begin{tabular}{|c|c|c|c|c|c|c|c|c|}
\hline \multirow{2}{*}{${ }^{{ }^{\circ} \mathrm{K}}$} & \multicolumn{2}{|c|}{$\frac{C_{p}^{0}}{R}$} & \multicolumn{2}{|c|}{$\frac{\left(H^{\circ}-E_{0}^{\circ}\right)}{R T}$} & \multicolumn{2}{|c|}{$\frac{-\left(F^{\circ}-E_{0}^{\circ}\right)}{R T}$} & $\frac{S}{H}$ & \\
\hline & 4. 00238 & 14 & 3. 96864 & 563 & 14.84410 & 72410 & 18. 81274 & 72974 \\
\hline 60 & 4.00252 & 22 & 3. 97427 & 405 & 15.56820 & 61295 & 19.54248 & 61700 \\
\hline 70 & 4. 00274 & 26 & 3. 97832 & 307 & 16.18115 & 53145 & 20.15948 & 53451 \\
\hline 80 & 4.00300 & 29 & 3. 98139 & 242 & 16. 71260 & 46908 & 20. 69399 & 47150 \\
\hline 90 & 4.00329 & 35 & 3. 98381 & 196 & 17. 18168 & 41984 & 21. 16549 & 42181 \\
\hline 100 & 4. 00364 & 46 & 3. 98577 & 165 & 17. 60152 & 37997 & 21. 58730 & 38161 \\
\hline 110 & 4. 00410 & 66 & 3. 98742 & 141 & 17. 98149 & 34701 & 21. 96891 & 34843 \\
\hline 120 & 4. 00476 & 101 & 3. 98883 & 126 & 18.32850 & 31933 & 22. 31734 & 32059 \\
\hline 130 & 4. 00577 & 154 & 3. 99009 & 118 & 18.64783 & 29574 & 22. 63793 & 29691 \\
\hline 140 & 4. 00731 & 224 & 3. 99127 & 114 & 18. 94357 & 27541 & 22. 93484 & 27655 \\
\hline 150 & 4. 00955 & 314 & 3. 99241 & 116 & 19. 21898 & 25770 & 23. 21139 & 25886 \\
\hline 160 & 4. 01269 & 419 & 3. 99357 & 124 & 19. 47668 & 24214 & 23.47025 & 24339 \\
\hline 170 & 4. 01688 & 537 & 3. 99481 & 137 & 19. 71882 & 22838 & 23. 71364 & 22975 \\
\hline 180 & 4. 02225 & 664 & 3. 99618 & 154 & 19. 94720 & 21610 & 23. 94339 & 21764 \\
\hline 190 & 4. 02889 & 797 & 3. 99772 & 175 & 20.16330 & 20510 & 24. 16103 & 20685 \\
\hline 200 & 4. 03686 & 930 & 3. 99947 & 200 & 20.36840 & 19519 & 24. 36788 & 19718 \\
\hline 210 & 4. 04616 & 1063 & 4. 00147 & 227 & 20. 56359 & 18620 & 24. 56506 & 18847 \\
\hline 220 & 4. 05679 & 1190 & 4. 00374 & 256 & 20. 74979 & 17802 & 24.75353 & 18059 \\
\hline 230 & 4. 06869 & 1313 & 4. 00630 & 287 & 20.92781 & 17057 & 24. 93412 & 17343 \\
\hline 240 & 4. 08182 & 1428 & 4. 00917 & 319 & 21. 09838 & 16373 & 25.10755 & 16692 \\
\hline 250 & 4. 09610 & 1535 & 4. 01236 & 351 & 21. 26211 & 15743 & 25. 27447 & 16095 \\
\hline 260 & 4. 11145 & 1634 & 4. 01587 & 384 & 21.41954 & 15163 & 25.43542 & 15547 \\
\hline 270 & 4. 12779 & 1726 & 4. 01971 & 417 & 21.57117 & 14627 & 25.59089 & 15043 \\
\hline 280 & 4. 14505 & 1808 & 4. 02388 & 448 & 21. 71744 & 14128 & 25. 74132 & 14576 \\
\hline 290 & 4. 16313 & 1884 & 4. 02836 & 481 & 21.85872 & 13664 & 25.88708 & 14146 \\
\hline 300 & 4. 18197 & 1853 & 4. 03317 & 511 & 21. 99536 & 13233 & 26.02854 & 13744 \\
\hline 310 & 4. 20150 & 2015 & 4. 03828 & 541 & 22.12769 & 12830 & 26. 16598 & 13371 \\
\hline 320 & 4. 22165 & 2072 & 4. 04369 & 571 & 22. 25599 & 12452 & 26. 29969 & 13022 \\
\hline 330 & 4. 24237 & 2123 & 4. 04940 & 599 & 22.38051 & 12097 & 26. 42991 & 12696 \\
\hline 340 & 4. 26360 & 2170 & 4. 05539 & 625 & 22.50148 & 11765 & 26.55687 & 12391 \\
\hline 350 & 4. 28530 & 2212 & 4. 06164 & 652 & 22. 61913 & 11451 & 26. 68078 & 12103 \\
\hline 360 & 4. 30742 & 2250 & 4. 06816 & 677 & 22.73364 & 11155 & 26.80181 & 11832 \\
\hline 370 & 4. 32992 & 2286 & 4. 07493 & 701 & 22.84519 & 10877 & 26. 92013 & 11578 \\
\hline 380 & 4. 33278 & 2316 & 4. 08194 & 724 & 22.95396 & 10612 & 27. 03591 & 11336 \\
\hline 390 & 4. 37594 & 2345 & 4. 08918 & 747 & 23.06008 & 10362 & 27.14927 & 11108 \\
\hline 400 & 4. : 9939 & 12066 & 4. 09665 & 4029 & 23. 16370 & 48480 & 27. 26035 & 52510 \\
\hline 450 & 4. 52005 & 12430 & 4. 13694 & 4451 & 23. 64850 & 43814 & 27.78545 & 48264 \\
\hline 500 & 4. 64435 & 12565 & 4. 18145 & 4779 & 24.08664 & 40075 & 28. 26809 & 44855 \\
\hline 550 & 4. 77000 & 12499 & 4. 22924 & 5028 & 24. 48739 & 37014 & 28.71664 & 42041 \\
\hline 600 & 4. 89499 & 12267 & 4. 27952 & 5208 & 24.85753 & 34459 & 29.13705 & 39668 \\
\hline 650 & 5.01766 & 11902 & 4. 33160 & 5328 & 25. 20212 & 32295 & 29. 52373 & 37623 \\
\hline 700 & 5. 13668 & 11438 & 4. 38488 & 5396 & 25. 52507 & 30436 & 29. 90996 & 35832 \\
\hline 750 & 5. 25106 & 10904 & 4. 43884 & 5420 & 25.82943 & 28821 & 30. 26828 & 34241 \\
\hline 800 & 5. 36010 & 10330 & 4. 49304 & 5407 & 26.11764 & 27401 & 30.61069 & 32808 \\
\hline 850 & 5. 46340 & 9736 & 4. 54711 & 5364 & 26. 39165 & 26143 & 30.93877 & 31506 \\
\hline 900 & 5.56076 & 9142 & 4. 60075 & 5296 & 26. 65308 & 25017 & 31. 25383 & 30314 \\
\hline 950 & 5. 65218 & 8557 & 4. 65371 & 5209 & 26.90325 & 24004 & 31.55697 & 29212 \\
\hline 1000 & 5. 73775 & 7993 & 4. 70580 & 5106 & 27. 14329 & 23083 & 31. 84909 & 28190 \\
\hline 1050 & 5.81768 & 7455 & 4. 75686 & 4993 & 27. 37412 & 22245 & 32. 13099 & 27238 \\
\hline 1100 & 5.89223 & 6945 & 4. 80679 & 4873 & 27. 59657 & 21475 & 32.40337 & 26347 \\
\hline 1150 & 5. 96168 & 6467 & 4. 85552 & 4745 & 27.81132 & 20765 & 32. 66684 & 25511 \\
\hline 1200 & 6.02635 & 11624 & 4. 90297 & 9099 & 28.01897 & 39608 & 32.92195 & 48706 \\
\hline 1300 & 6. 14259 & 10082 & 4. 99396 & 8573 & 28.41505 & 37326 & 33. 40901 & 45900 \\
\hline 1400 & 6. 24341 & 8761 & 5. 07969 & 8057 & 28.78831 & 35324 & 33. 86801 & 43381 \\
\hline 1500 & 6. 33102 & 7636 & 5. 16026 & 7561 & 29.14155 & 33548 & 34. 30182 & 41108 \\
\hline 1600 & 6. 40738 & 6680 & 5. 23587 & 7092 & 29.47703 & 31957 & 34. 71290 & 39050 \\
\hline 1700 & 6.47418 & 5864 & 5. 30679 & 6652 & 29. 79660 & 30523 & 35.10340 & 37175 \\
\hline 1800 & 6. 53282 & 5168 & 5. 37331 & 6242 & 30.10183 & 29221 & 35.47515 & 35462 \\
\hline 1900 & 6. 58450 & 4572 & 5. 43573 & 5860 & 30.39404 & 28033 & 35.82977 & 33893 \\
\hline 2000 & 6. 63022 & 4059 & 5. 49433 & 5508 & 30.67437 & 26941 & 36.16870 & 32449 \\
\hline 2100 & 6. 67081 & 3617 & 5. 54941 & 5181 & 30.94378 & 25937 & 36.49319 & 31118 \\
\hline 2200 & 6. 70698 & 3233 & 5. 60122 & 4879 & 31. 20315 & 25007 & 36.80437 & 29886 \\
\hline 2300 & 6. 73931 & 2898 & 5. 65001 & 4600 & 31.45322 & 24144 & 37. 10323 & 28745 \\
\hline 2400 & 6. 76829 & 2605 & 5. 69601 & 4342 & 31. 69466 & 23341 & 37. 39068 & 27683 \\
\hline 2500 & 6. 79434 & 2346 & 5. 73943 & 4103 & 31.92807 & 22592 & 37. 66751 & 26695 \\
\hline 2600 & 6.81780 & 2119 & 5. 78046 & 3882 & 32.15399 & 21889 & 37. 93446 & 25771 \\
\hline 2700 & 6.83899 & 1916 & 5. 81928 & 3677 & 32.37288 & 21230 & 35. 19217 & 24907 \\
\hline 2800 & 6. 85815 & 1735 & 5.85605 & 3486 & 32.58518 & 20611 & 38. 44124 & 24097 \\
\hline 2900 & 6.87550 & 1573 & 5. 89091 & 3308 & 32. 79129 & 20028 & 38. 68221 & 23336 \\
\hline 3000 & 6.89123 & 2720 & 5. 92399 & 6133 & 32.99157 & 38431 & 38.91557 & 44564 \\
\hline 3200 & 6. 91843 & 2236 & 5. 98532 & 5557 & 33.37588 & 36456 & 39. 36121 & 42012 \\
\hline 3400 & & 1830 & & 5052 & 33. 74044 & 34674 & 39. 78133 & 39726 \\
\hline 3600 & 6. 95909 & 1483 & 6. 09141 & 4607 & 34.08718 & 33060 & 40.17859 & 37667 \\
\hline 3800 & 6. 97392 & 1180 & 6. 13748 & 4213 & 34. 41778 & 31589 & 40.55526 & 35803 \\
\hline 4000 & 6. 98572 & 913 & 6. 17961 & 3862 & 34.73367 & 30246 & 40.91329 & 34107 \\
\hline 4200 & 6. 99485 & 673 & 6. 21823 & 3546 & 35.03613 & 29010 & 41. 25436 & 32556 \\
\hline 4400 & 7. 00158 & 454 & 6. 25369 & 3262 & 35.32623 & 27872 & 41. 57992 & 31134 \\
\hline 4600 & 7. 00612 & 250 & 6. 28631 & 3005 & 35.60495 & 26818 & 41.89126 & 29824 \\
\hline 4800 & 7. 00862 & 60 & 6. 31636 & 2771 & 35.87313 & 25842 & 42.18950 & 28613 \\
\hline 5000 & 7. 00922 & -... & 6. 34407 & $\ldots$ & 36.13155 & -.... & 42.47563 & .... \\
\hline
\end{tabular}


TABLE 5. Ideal gas thermodynamic functions for HTS

\begin{tabular}{|c|c|c|c|c|c|c|c|c|}
\hline \multirow{2}{*}{${ }^{{ }^{\circ} \mathrm{K}}$} & \multicolumn{2}{|c|}{$\frac{C_{p}^{\circ}}{R}$} & \multicolumn{2}{|c|}{$\frac{\left(H^{\circ}-E_{0}^{\circ}\right)}{R T}$} & \multicolumn{2}{|c|}{$\frac{-\left(F^{\circ}-E_{0}^{\circ}\right)}{R T}$} & $\frac{S}{I}$ & \\
\hline & 4. 00211 & 22 & 3. 97622 & 433 & 14. 72545 & 72535 & 18. 70167 & 72969 \\
\hline 60 & 4. 00233 & 26 & 3. 98055 & 313 & 15. 45080 & 61386 & 19. 43136 & 61699 \\
\hline 70 & 4. 00259 & 30 & 3. 98368 & 239 & 16. 06466 & 53211 & 20. 04835 & 53449 \\
\hline 80 & 4. 00289 & 33 & 3.98607 & 188 & 16.59677 & 46960 & 20.58284 & 47149 \\
\hline 90 & 4. 00322 & 41 & 3. 98795 & 155 & 17. 06637 & 42026 & 21.05433 & 42180 \\
\hline 100 & 4. 00363 & 59 & 3.98950 & 131 & 17. 48663 & 38030 & 21.47613 & 38161 \\
\hline 110 & 4. 00422 & 92 & 3. 99081 & 115 & 17. 86693 & 34730 & 21.85774 & 34845 \\
\hline 120 & 4. 00514 & 144 & 3. 99196 & 107 & 18. 21423 & 31957 & 22.20619 & 32664 \\
\hline 130 & 4. 00658 & 220 & 3. 99303 & 104 & 18. 53380 & 29595 & 22.52683 & 29699 \\
\hline 140 & 4. 00878 & 316 & 3. 99407 & 108 & 18.82975 & 27560 & 22.82382 & 27668 \\
\hline 150 & 4. 01194 & 437 & 3.99515 & 118 & 19. 10535 & 25788 & 23.10050 & 25906 \\
\hline 160 & 4. 01631 & 574 & 3.99633 & 134 & 19.36323 & 24231 & 23.35956 & 24365 \\
\hline 170 & 4. 02205 & 725 & 3. 99767 & 154 & 19.60554 & 22855 & 23.60321 & 23010 \\
\hline 180 & 4. 02930 & 886 & 3.99921 & 181 & 19. 83409 & 21627 & 23.83331 & 21808 \\
\hline 190 & 4. 03816 & 1051 & 4. 00102 & 212 & 20.05036 & 20528 & 24. 05139 & 20739 \\
\hline 200 & 4. 04867 & 1217 & 4. 00314 & 245 & 20.25564 & 19537 & 24. 25878 & 19782 \\
\hline 210 & 4. 06084 & 1381 & 4. 00559 & 282 & 20. 45101 & 18640 & 24.45660 & 18923 \\
\hline 220 & 4.07465 & 1540 & 4. 00841 & 321 & 20.63741 & 17825 & 24. 64583 & 18146 \\
\hline 230 & 4. 09005 & 1691 & 4. 01162 & 361 & 20.81566 & 17081 & 24. 82729 & 17442 \\
\hline 240 & 4. 10696 & 1834 & 4. 01523 & 403 & 20.98647 & 16399 & 25.00171 & 16802 \\
\hline 250 & 4.12530 & 1967 & 4. 01926 & 446 & 21.15046 & 15773 & 25. 16973 & 16218 \\
\hline 260 & 4. 14497 & 2090 & & 487 & 21.3 & 15194 & 3191 & 15682 \\
\hline 270 & 4. 16587 & 2203 & 4. 02859 & 529 & 21.46013 & 14661 & 25.48873 & 15190 \\
\hline 280 & 4. 18790 & 2304 & 4. 03388 & 571 & 21. 60674 & 14165 & 25. 64063 & 14735 \\
\hline 290 & 4. 21094 & 2395 & 4. 03959 & 611 & 21.74839 & 13705 & 25.78798 & 14316 \\
\hline 300 & 4. 23489 & 2477 & 4. 04570 & 650 & 21.88544 & 13276 & 25. 93114 & 13927 \\
\hline 310 & 4. 25966 & 2549 & 4. 05220 & 688 & 22.01820 & 12876 & 26. 07041 & 13564 \\
\hline 320 & 4. 28515 & 2611 & 4. 05908 & 724 & 22.14696 & 12502 & 26. 20605 & 13226 \\
\hline 330 & 4. 31126 & 2666 & 4. 06632 & 760 & 22. 27198 & 12150 & 26. 33831 & 12909 \\
\hline 340 & 4. 33792 & 2712 & 4. 07392 & 793 & 22.39348 & 11821 & 26.46740 & 12614 \\
\hline 350 & 4. 36504 & 2751 & 4. 08185 & 824 & 22.51169 & 11510 & 26.59354 & 12335 \\
\hline 360 & 4. 39255 & 2784 & & 855 & & 11218 & 689 & 12073 \\
\hline 370 & 4. 42039 & 2810 & 4. 09864 & 884 & 22. 73897 & 10942 & 26.83762 & 11826 \\
\hline 380 & 4. 44849 & 2831 & 4. 16748 & 911 & 22.84839 & 10681 & 26. 95588 & 11592 \\
\hline 390 & 4. 47680 & 2846 & 4. 11659 & 936 & 22.95520 & 10435 & 27.07180 & 11370 \\
\hline 400 & 4. 50526 & 14317 & 4. 12595 & 5009 & 23.05955 & 48881 & $27.18 巨 50$ & 53890 \\
\hline 450 & 4. 64843 & 14172 & 4. 17604 & 5435 & 23.54836 & 44277 & 27. 72440 & 49713 \\
\hline 500 & 4. 79015 & 13771 & 4. 23039 & 5719 & 23. 99113 & 405 & 28.22153 & 46304 \\
\hline 550 & 4. 92786 & 13220 & 4. 28758 & 5890 & 24. 39699 & 37559 & 28. 68457 & 43450 \\
\hline 600 & 5. 06006 & 12583 & 4. 34648 & 5978 & 24. 77258 & 35026 & 29.11907 & 41003 \\
\hline 650 & 5. 18589 & 11902 & 4. 40626 & 5998 & 25.12284 & 32873 & 29.52910 & 38872 \\
\hline 700 & 5. 30491 & 11203 & 4. 46624 & 5968 & 25. & 310 & & 36986 \\
\hline 750 & 5. 41694 & 10503 & 4. 52592 & 5901 & 25.76175 & $\begin{array}{l}29399 \\
2930\end{array}$ & 30.28768 & $\begin{array}{l}35290 \\
35290\end{array}$ \\
\hline 800 & 5. 52197 & 9817 & 4. 58493 & 5804 & 26.05574 & 27970 & 30.64 & 33775 \\
\hline 850 & 5. 60214 & 9154 & 4. 64297 & $\begin{array}{l}56086 \\
5686\end{array}$ & 26. 33544 & 26700 & 30.97842 & 32386 \\
\hline 900 & 5. 71168 & 8521 & 4. 69983 & 5553 & & 25560 & 28 & 31112 \\
\hline 950 & 5. 7968 & 7920 & 4. 75536 & 5408 & 26.85804 & 24530 & 31.61340 & 26938 \\
\hline 1000 & 5. 87609 & $7 \leq 56$ & 4. 80944 & 5256 & 27.10334 & 23594 & 31. 91278 & 28850 \\
\hline 1050 & 5. 94965 & 6829 & 4.86200 & 5101 & 27. 33928 & 22736 & 32.20128 & 27838 \\
\hline 1100 & 6. 01794 & 6339 & 4. 91301 & 4944 & 27. 56664 & 21949 & 32.47966 & $\begin{array}{l}26890 \\
2689\end{array}$ \\
\hline 1150 & 6. 08133 & 5885 & 4. 96245 & 4786 & 27. 78613 & 21221 & 32.74858 & 26008 \\
\hline 1200 & 6. 14018 & 10542 & 5.01031 & 9107 & 27. 99834 & 40468 & 33.00866 & 49574 \\
\hline & & 9112 & & & & & 0440 & 46627 \\
\hline 1400 & 6. 33672 & 7902 & 5. 18644 & 7938 & & 36057 & 33.97067 & 43994 \\
\hline 150 & 6. 41574 & 6878 & 5. 26582 & 74 & & 34224 & 34. 41061 & 41631 \\
\hline 1600 & 6.48452 & 6011 & 5. 33989 & 6914 & 29.48703 & 32583 & 34.82692 & 39497 \\
\hline 1700 & 6. 54463 & 5275 & 5. 40903 & 6458 & 29.81286 & 31102 & 35.22189 & 37560 \\
\hline & & & & & & & & 35798 \\
\hline 1900 & 6. 64387 & 4113 & 5. 53401 & 5654 & 30.42146 & 28 & 35. 95547 & 34185 \\
\hline 2000 & & 3652 & 5. 59055 & 5300 & 30.70677 & 27406 & 36. 29732 & 32707 \\
\hline 2100 & 6.72152 & 3255 & 5. 64355 & 4975 & 30.98083 & 26370 & 36.62439 & 31345 \\
\hline 2200 & 6. 75407 & 2910 & 5. 69330 & 4677 & 31.24453 & 25412 & 36.93784 & 30088 \\
\hline & & & & & & & & 28925 \\
\hline 2400 & 6.80927 & 2347 & & & & 236 & 37.52797 & 27845 \\
\hline 2500 & & 2115 & & & & 229 & 37.80 & 26841 \\
\hline 2600 & 6. 85389 & 1909 & 5.86472 & 3700 & 32.21010 & 22204 & 38.07483 & $\begin{array}{l}20041 \\
25903\end{array}$ \\
\hline 2700 & 6.87298 & 1727 & 5. 90172 & 3500 & 32.43214 & 21527 & 38.33386 & 25027 \\
\hline & & & & & & & & 24207 \\
\hline 2900 & & 1417 & & 3144 & & 20292 & 38.826 & 23436 \\
\hline 3000 & 6. 92006 & 2449 & 6. 00131 & 5821 & 33.05924 & 38921 & 39.06056 & 44742 \\
\hline 3200 & 6.94455 & 2008 & 6. 05952 & 5268 & 33.44845 & 36896 & 39.50798 & 42163 \\
\hline & & & & & & & & \\
\hline & 100 & 131 & 002 & 43 & 34. & 334 & 40.32818 & 37781 \\
\hline 380 & & 1038 & 6. 20359 & 3980 & 34. 50239 & 31923 & 40. 70599 & 35903 \\
\hline 4000 & 7. 00456 & 790 & 6. 24339 & 3645 & 34. 82162 & 30551 & 41.06502 & 34195 \\
\hline 4200 & 7. 01246 & 564 & 6. 27984 & 3343 & 35.12713 & 29292 & 41. 40697 & 32636 \\
\hline & & 356 & & 3073 & 35.42005 & 28133 & 41. 73333 & 31206 \\
\hline & & 164 & 6.34400 & 2828 & 35.70138 & 27060 & 42.04539 & 29888 \\
\hline 4800 & 7. 02330 & -20 & 6. 37228 & 2604 & 35. 97198 & 26067 & 42.34427 & 28670 \\
\hline 5000 & 7. 02310 & - . & 6. 39832 & --.- & 36. 23265 & & 42. 63097 & ..... \\
\hline
\end{tabular}

TABLE 6. Ideal gas thermodynamic functions for $\mathrm{D}_{2} \mathrm{~S}$

\begin{tabular}{|c|c|c|c|c|c|c|c|c|}
\hline \multirow{2}{*}{$\frac{{ }^{\circ} \mathrm{K}}{50}$} & \multicolumn{2}{|c|}{$\frac{C_{p}^{\circ}}{R}$} & \multicolumn{2}{|c|}{$\frac{\left(H^{\circ}-E_{0}^{\circ}\right)}{R T}$} & \multicolumn{2}{|c|}{$\frac{-\left(F^{\circ}-E_{0}^{\circ}\right)}{R T}$} & $\frac{S}{H}$ & \\
\hline & 4. 00228 & 17 & 3. 97643 & 432 & 15. 20882 & 72540 & 19.18526 & 72972 \\
\hline 60 & 4. 00245 & 24 & 3.98075 & 312 & 15. 93422 & 61388 & 19. 91498 & 61700 \\
\hline 70 & 4. 00269 & 30 & 3. 98387 & 237 & 16.54810 & 53213 & 20.53198 & 53450 \\
\hline 80 & 4. 00299 & 46 & 3. 98624 & 189 & 17.08023 & 46963 & 21.06648 & 47151 \\
\hline 90 & 4. 00345 & 75 & 3.98813 & 156 & 17. 54986 & 42027 & 21. 53799 & 42184 \\
\hline 100 & 4. 00420 & 133 & 3. 98969 & 138 & 17. 97013 & 38033 & 21. 95983 & 38170 \\
\hline 110 & 4. 00553 & 222 & 3. 99107 & 129 & 18. 35046 & 34732 & 22.34153 & 34861 \\
\hline 120 & 4. 00775 & 346 & 3.99236 & 130 & 18. 69778 & 31961 & 22. 69014 & 32092 \\
\hline 130 & 4. 01121 & 500 & 3. 99366 & 143 & 19.01739 & 29602 & 23.01106 & 29744 \\
\hline 140 & 4. 01621 & 678 & 3.99509 & 162 & 19. 31341 & 27568 & 23.30850 & 27731 \\
\hline 150 & 4. 02299 & 871 & 3. 99671 & 190 & 19. 58909 & 25800 & 23.58581 & 25990 \\
\hline 160 & 4. 03170 & 1068 & 3. 99861 & 226 & 19. 84709 & 24248 & 23.84571 & 24473 \\
\hline 170 & 4. 04238 & 1263 & 4. 00087 & 264 & 20.08957 & 22876 & 24. 09044 & 23141 \\
\hline 180 & 4. 05501 & 1447 & 4. 00351 & 309 & 20.31833 & 21654 & 24.32185 & 21962 \\
\hline 190 & 4. 06948 & 1620 & 4. 00660 & 354 & 20.53487 & 20560 & 24.54147 & 20914 \\
\hline 200 & 4. 08568 & 1775 & 4. 01014 & 401 & 20. 74047 & 19575 & 24. 75061 & 19977 \\
\hline 210 & 4. 10343 & 1914 & 4. 01415 & 449 & 20.93622 & 18684 & 24. 95038 & 19133 \\
\hline 220 & 4. 12257 & 2036 & 4. 01864 & 496 & 21. 12306 & 17874 & 25. 14171 & 18370 \\
\hline 230 & 4. 14293 & 2142 & 4. 02360 & 541 & 21.30180 & 17136 & 25.32541 & 17677 \\
\hline 240 & 4. 16435 & 2235 & 4. 02901 & 586 & 21. 47316 & 16459 & 25.50218 & 17045 \\
\hline 250 & 4. 18670 & 2316 & 4. 03487 & 628 & 21. 63775 & 15837 & 25.67263 & 16465 \\
\hline 260 & 4. 20986 & 2387 & 4. 04115 & 669 & 21. 79612 & 15264 & 25. 83728 & 15933 \\
\hline 270 & 4. 23373 & 2451 & 4. 04784 & 708 & 21. 94876 & 14734 & 25. 99661 & 15441 \\
\hline 280 & 4. 25824 & 2507 & 4. 05492 & 744 & 22.09610 & 14242 & 26.15102 & 14986 \\
\hline 290 & 4. 28331 & 2558 & 4. 06236 & 779 & 22. 23852 & 13785 & 26. 30088 & 14564 \\
\hline 300 & 4. 30889 & 2606 & 4. 07015 & 812 & 22. 37637 & 13359 & 26. 44652 & 14172 \\
\hline 310 & 4. 33495 & 2650 & 4. 07827 & 843 & 22. 50996 & 12961 & 26.58824 & 13804 \\
\hline 320 & 4. 36145 & 2692 & 4. 08670 & 874 & 22. 63957 & 12589 & 26. 72628 & 13462 \\
\hline 330 & 4. 38837 & 2732 & 4. 09544 & 901 & 22. 76546 & 12240 & 26.86090 & 13141 \\
\hline 340 & 4. 41569 & 2769 & 4. 10445 & 929 & 22. 88786 & 11911 & 26. 99231 & 12840 \\
\hline 350 & 4. 44338 & 2806 & 4. 11374 & 955 & 23. 00697 & 11602 & 27.12071 & 12557 \\
\hline 360 & 4. 47144 & 2841 & 4. 12329 & 979 & 23. 12299 & 11311 & 4628 & 12290 \\
\hline 370 & 4. 49985 & 2873 & 4. 13308 & 1003 & 23. 23610 & 11035 & 27. 36918 & 12038 \\
\hline 380 & 4. 52858 & 2904 & 4. 14311 & 1025 & 23. 34645 & 10775 & 27. 48956 & 11801 \\
\hline 390 & 4. 55762 & 2933 & 4. 15336 & 1048 & 23.45420 & 10529 & 27.60757 & 11576 \\
\hline 400 & 4. 58695 & 15015 & 4. 16384 & 5530 & 23. 55949 & 49357 & 27. 72333 & 54888 \\
\hline 450 & 4. 73710 & 15355 & 4. 21914 & 5946 & 24. 05306 & 44758 & 28. 27221 & 50704 \\
\hline 500 & 4. 89065 & 15348 & 4. 27860 & 6263 & 24. 50064 & 41071 & 28.77925 & 47334 \\
\hline 550 & 5. 04413 & 15028 & 4. 34123 & 6487 & 24. 91135 & 38051 & 29. 25259 & 44537 \\
\hline 600 & 5. 19441 & 14464 & 4. 40610 & 6624 & 25. 29186 & 35528 & 29. 69796 & 42153 \\
\hline 650 & 5. 33905 & 13739 & 4. 47234 & 6687 & 25. 64714 & 33389 & 30.11949 & 40075 \\
\hline 700 & 5,47644 & 12919 & 4. 53921 & 6683 & 25. 98103 & 31545 & 024 & 38229 \\
\hline 750 & 5. 60563 & 12060 & 4. 60604 & 6629 & 26. 29648 & 29939 & 30.90253 & 36567 \\
\hline 800 & 5. 72623 & 11202 & 4. 67233 & 6533 & 26. 59587 & 28522 & 31. 26820 & 35056 \\
\hline 850 & 5. 83825 & 10369 & 4. 73766 & 6406 & 26. 88109 & 27262 & 31.61876 & 33668 \\
\hline 900 & 5. 94194 & 9581 & 4. 80172 & 6257 & 27. 15371 & 26130 & 31.95544 & 32386 \\
\hline 950 & 6. 03775 & 8844 & 4. 86429 & 6091 & 27. 41501 & 25106 & 32. 27930 & 31198 \\
\hline 1000 & 6. 12619 & 8163 & 4. 92520 & 5916 & 27. 66607 & 24174 & 32.59128 & 30090 \\
\hline 1050 & 6. 20782 & 7538 & 4. 98436 & 5735 & 27. 90781 & 23320 & 32.89218 & 29055 \\
\hline 1100 & 6. 28320 & 6967 & 5. 04171 & 5551 & 28. 14101 & 22535 & 33.18273 & 28086 \\
\hline 1150 & 6. 35287 & 6449 & 5. 09722 & 5368 & 28. 36636 & 21808 & 33.46359 & 27175 \\
\hline 1200 & 6. 41736 & 11527 & 5. 15090 & 10196 & 28.58444 & 41636 & 33. 73534 & 51833 \\
\hline 1300 & & 9977 & 5. 25286 & 9506 & & 39 & 34. 25367 & 48786 \\
\hline 1400 & 6. 63240 & 8702 & 5. 34792 & 8860 & 29. 39360 & 37203 & 34. 74153 & 46062 \\
\hline 1500 & 6. 71942 & 7654 & 5. 43652 & 8262 & 29. 76563 & 35353 & 35. 20215 & 43616 \\
\hline 1600 & 6. 79596 & 6787 & 5. 51914 & 7714 & 30.11916 & 33694 & 35.63831 & 41408 \\
\hline 1700 & 6.86383 & 6065 & 5. 59628 & 7214 & 30.45610 & 32194 & 36.05239 & 39407 \\
\hline 1800 & 6. 92448 & & 5. 66842 & 6757 & & & & 37588 \\
\hline 1900 & 6.97910 & 4953 & 5. 73599 & 6341 & 31.08634 & 29585 & 36.82234 & 35926 \\
\hline 2000 & 7.02863 & 4522 & 5. 79940 & 5963 & 31.38219 & 28441 & 37.18160 & 34403 \\
\hline 2100 & 7. 07385 & 4155 & 5. 85903 & 5617 & 31. 66660 & 27387 & 37.52563 & 33005 \\
\hline 2200 & 7. 11540 & 3839 & 5. 91520 & 5303 & 31. 94047 & 26413 & 37.85568 & 31715 \\
\hline & & & & 5015 & & & & 30523 \\
\hline 2400 & 7. 18945 & 3330 & 6. 01838 & 4752 & 32. 45967 & 24666 & 38.47806 & 29417 \\
\hline 2500 & 7. 22275 & 3123 & 6.06590 & 4510 & 32. 70633 & 23879 & 38. 77223 & 28389 \\
\hline 2600 & 7. 25398 & 2940 & 6. 11100 & 4288 & 32.94512 & 23144 & 39.05612 & 27433 \\
\hline 2700 & 7. 28338 & 2780 & 6.15388 & 4084 & 33.17656 & 22455 & 39.33045 & 26539 \\
\hline & & & & & & & & 25702 \\
\hline 2900 & 7. 33755 & 2510 & 6. 23368 & 3722 & 33. 61918 & 21196 & 39.85286 & 24918 \\
\hline 3000 & 7. 36265 & 4686 & 6. 27090 & 6972 & 33.83114 & 40697 & 40.10204 & 47669 \\
\hline 3200 & 7. 40951 & 4311 & 6. 34062 & 6416 & 34. 23811 & 38635 & 40.57873 & 45051 \\
\hline & & & & & & & & \\
\hline 3600 & 7. 49260 & 3735 & 6.46411 & 5513 & 34.99225 & 35099 & 41.45637 & 40611 \\
\hline 3800 & 7. 52995 & 3509 & 6.51924 & 5142 & 35.34324 & 33572 & 41. 86248 & 38714 \\
\hline 4000 & 7. 56504 & 3312 & 6.57066 & 4815 & 35.67896 & 32176 & 42. 24962 & 36991 \\
\hline 4200 & 7. 59816 & 3138 & 6. 61881 & 4523 & 36.00072 & 30896 & 42,61953 & 35420 \\
\hline 4400 & 7. 62954 & 2983 & 6. 66404 & 4264 & 36. 30968 & 29718 & 42. 97373 & 33981 \\
\hline 4600 & & 2843 & 6. 70668 & 4029 & 36.60686 & 28629 & 43. 31354 & 32658 \\
\hline 4800 & 7. 68780 & 2716 & 6. 74697 & 3818 & 36.89315 & 27621 & 43.64012 & 31439 \\
\hline 5000 & 7. 71496 & $\ldots$ & 6.78515 & .... & 37.16936 & 2.00 & 43. 95451 & - \\
\hline
\end{tabular}


TABLE 7. Ideal gas thermodynamic functions for DTS

\begin{tabular}{|c|c|c|c|c|c|c|c|c|}
\hline \multirow{2}{*}{${ }^{{ }^{\circ} \mathrm{K}}$} & \multicolumn{2}{|c|}{$\frac{C_{p}^{\circ}}{R}$} & \multicolumn{2}{|c|}{$\frac{\left(H^{\circ}-E_{0}^{\circ}\right)}{R T}$} & \multicolumn{2}{|c|}{$\frac{-\left(F^{\circ}-E_{0}^{\circ}\right)}{R T}$} & $\frac{S}{I}$ & \\
\hline & 4.00197 & 26 & 3. 98122 & 348 & 15.67893 & 72619 & 19. 66016 & 72966 \\
\hline 60 & 4. 00223 & 31 & 3. 98470 & 253 & 16. 40512 & 61444 & 20.38982 & 61697 \\
\hline 70 & 4. 00254 & 41 & 3. 98723 & 193 & 17.01956 & 53255 & 21.00679 & 53449 \\
\hline 80 & 4. 00295 & 68 & 3. 98916 & 157 & 17.55211 & 46995 & 21. 54128 & \\
\hline 90 & 4. 00363 & 124 & 3. 99073 & 135 & 18.02206 & 42054 & 22.01280 & 42188 \\
\hline 100 & 4.00487 & 220 & 3. 99208 & 125 & 18.44260 & 38055 & 22.43468 & 38180 \\
\hline 110 & 4.00707 & 358 & 3. 99333 & 128 & 18.82315 & 34752 & 22.81648 & 34880 \\
\hline 120 & 4. 01065 & 536 & 3. 99461 & 143 & 19.17067 & 31979 & 23.16528 & 32123 \\
\hline 130 & 4. 01601 & 741 & 3. 99604 & 168 & 19.49046 & 29620 & 23.48651 & 29787 \\
\hline 140 & 4. 02342 & 965 & 3. 99772 & 202 & 19. 78666 & 27588 & 23.78438 & 27791 \\
\hline 150 & 4. 03307 & 1193 & 3. 99974 & 244 & 20.06254 & 25821 & 24. 06229 & 26065 \\
\hline 160 & 4.04500 & 1416 & & & 20.32075 & 24272 & 24. 32294 & 24564 \\
\hline 170 & 4.05916 & 1628 & 4. 00511 & 344 & 20.56347 & 22902 & 24. 56858 & 23247 \\
\hline 180 & 4. 07544 & 1820 & 4. 00855 & 400 & 20. 79249 & 21684 & 24.80105 & 22083 \\
\hline 190 & 4. 09364 & 1993 & 4. 01255 & 454 & 21.00933 & 20593 & 25.02188 & 21047 \\
\hline 200 & 4.11357 & 2145 & 4.01709 & 510 & 21. 21526 & 19611 & 25. 23235 & 20122 \\
\hline 210 & 4. 13502 & 2279 & 4. 02219 & 564 & 21.41137 & 18724 & 25. 43357 & \\
\hline 220 & 4. 15781 & 2395 & 4. 02783 & & 21. 59861 & 17918 & 25. 62645 & 18535 \\
\hline 230 & 4. 18176 & 2495 & 4. 03400 & 667 & 21. 77779 & 17183 & 25. 81180 & 17850 \\
\hline 240 & 4. 20671 & 2581 & 4. 04067 & 716 & 21.94962 & 16509 & 25.99030 & 17224 \\
\hline 250 & 4. 23252 & 2657 & 4. 04783 & 761 & 22.11471 & 15891 & 26.16254 & 16652 \\
\hline 260 & 4. 25909 & 2724 & & & & & 26.32906 & 16125 \\
\hline 270 & 4. 28633 & 2785 & 4. 06349 & 84 & 22.42682 & 14793 & 26. 49031 & 15639 \\
\hline 280 & 4. 31418 & 2837 & 4. 07194 & 884 & & 14304 & 26. 64670 & 15188 \\
\hline 290 & 4. 34255 & 2885 & 4. 08078 & 921 & 22.71779 & 13850 & 26.79858 & 14771 \\
\hline 300 & 4. 37140 & 2930 & 4. 08999 & 955 & 22.85629 & 13427 & 26. 94629 & 14381 \\
\hline 310 & 4. 40070 & 2970 & 4. 099 & & 22.99056 & 13031 & 27.09010 & \\
\hline 320 & 4. 43040 & 3007 & 4. 10941 & 1018 & 23.12087 & 12661 & 27. 23028 & 13679 \\
\hline 330 & 4. 46047 & 3041 & 4. 11959 & 1048 & 23. 24748 & 12313 & 27. 36707 & 13361 \\
\hline 340 & 4. 49088 & 3072 & 4. 13007 & 1074 & 23.37061 & 11988 & 27.50068 & 13063 \\
\hline 350 & 4. 52160 & 3099 & 4. 14081 & 1101 & 23.49049 & 11680 & 27.63131 & 12781 \\
\hline 360 & 4. 55259 & 3124 & 182 & 1125 & 0729 & & 75912 & \\
\hline 370 & 4. 58383 & 3146 & 4. 16307 & 1149 & 23.72120 & 11117 & 27.88428 & 12266 \\
\hline 380 & 4. 61529 & 3164 & 4. 17456 & 1170 & 23.83237 & 10859 & 28.00694 & 12029 \\
\hline 390 & 4. 64693 & 3178 & 4. 18626 & 1192 & 23.94096 & 10614 & 28.12723 & 11805 \\
\hline 400 & 4. 67871 & 16001 & 4. 19818 & 6227 & 24.04710 & 49803 & 28. 24528 & 56031 \\
\hline & 4. 83872 & 15883 & 4. 26045 & & 24.54513 & 45226 & 0559 & \\
\hline 500 & 4. 99755 & 15410 & 4. 32625 & 680 & 24. 99739 & 41552 & 2364 & 483 \\
\hline 550 & 5. 15165 & 14673 & 4. 39433 & 6928 & 25. 41291 & 38532 & 29.80724 & 45460 \\
\hline 600 & 5. 29838 & 13771 & 4. 46361 & 6957 & 25. 79823 & 36003 & 30.26184 & 42960 \\
\hline 650 & 5. 43609 & 12783 & 4. 53318 & 6912 & 26.15826 & 33848 & 30.69144 & 40760 \\
\hline & 5. 56392 & 1177 & & & 9674 & 31985 & 904 & 38 \\
\hline 750 & 5. 68165 & 10785 & 4. 67039 & 6662 & 26.81659 & 30356 & 31.48698 & 370 \\
\hline 80 & 5. 78950 & 9846 & 4. 73701 & 6485 & 27.12015 & 28913 & 31.85716 & 35399 \\
\hline 850 & 5.88796 & 8968 & 4. 80186 & 6287 & 27.40928 & 27626 & 32.21115 & 33913 \\
\hline 900 & 5. 97764 & 8162 & 4. 86473 & 6076 & 27.68554 & 26466 & 32.55028 & 32541 \\
\hline & & 742 & 4. 92549 & 58 & & 2541 & 32.87569 & 31272 \\
\hline 1000 & 6.13351 & 6759 & 4. 98406 & 5637 & 28. 2043 & 24455 & 33. 18841 & 30092 \\
\hline 1050 & 6. 20110 & 6157 & 5. 04043 & 5418 & 28.44889 & 23574 & 33.48933 & \\
\hline 1100 & 6.26267 & 5615 & 5.09461 & 5202 & 28.68463 & 22762 & 33.77924 & 27965 \\
\hline 1150 & 6.31882 & 5130 & 5. 14663 & 4993 & 28.91225 & 22010 & 34.05889 & 27003 \\
\hline 1200 & 6. 37012 & 8992 & 5. $1965 f$ & 938 & & & 34. 32892 & 51353 \\
\hline & 46004 & 757 & & & 29. 55205 & & & \\
\hline 140 & .53582 & 6432 & 5. 37672 & 794 & 29.94732 & 373 & 32404 & 45318 \\
\hline 150 & 6.60014 & 5500 & 5. 45620 & 732 & 30.32102 & 35451 & 35. 77722 & 42777 \\
\hline 1600 & 6.65514 & 4736 & 5.52945 & 6765 & 30.67553 & 33728 & 36. 20499 & 40492 \\
\hline 1700 & 6. 70250 & 4104 & 5. 59710 & 6258 & 31.01281 & 32171 & 36. 60991 & 38429 \\
\hline & & $35 \%$ & & & & & & \\
\hline 190 & 6.77933 & 313 & 5.717 & & 31.642 & & 37. 35979 & \\
\hline 200 & 6.81072 & 2768 & & & 31.936 & & 37. 70834 & 33298 \\
\hline 2100 & 6.83840 & 2451 & 5.82172 & 4678 & 32.21959 & 27192 & 38.04132 & 31870 \\
\hline 2200 & 6. 86291 & 2180 & 5.86850 & 4372 & 32. 49151 & 261 & & \\
\hline & & 1946 & & & & & & \\
\hline & 90417 & 1743 & & & 33.005 & & 6901 & 28220 \\
\hline 250 & 6. 92160 & 1565 & 5. 99155 & 360 & 33. 24965 & & & \\
\hline 2600 & 6.93725 & 1408 & 6.02763 & 3395 & 33.48535 & 22813 & 39.51299 & 26208 \\
\hline 2700 & 6.95133 & 1269 & & & 33. 71348 & 22103 & 39. 77507 & 25304 \\
\hline & & & & & & & & \\
\hline 290 & 6.97547 & 1034 & 6.12381 & 2856 & 34.14888 & 20809 & 40. 27269 & \\
\hline 3000 & 6.98581 & 1774 & 6.15237 & 5266 & 34.35697 & 39878 & 40.50934 & 45144 \\
\hline 3200 & 7. 00355 & 1439 & 6. 20503 & 4741 & & & 40. 96078 & 42504 \\
\hline & & 1153 & & & & & & \\
\hline & 7. 02947 & 905 & & & & & & \\
\hline & & 68 & 6.334 & & & & 42. 16761 & 36121 \\
\hline 4000 & 7.04537 & 486 & 6. 36959 & 3230 & 36.15923 & 31157 & 42.52882 & 34387 \\
\hline 4200 & 7. 05023 & 304 & 6.40189 & 2955 & 36.47080 & 29850 & 42.87269 & 32806 \\
\hline & 7. 05327 & 133 & & & & & & \\
\hline & 7.05460 & -29 & 6.45851 & 2483 & 37.05580 & 27540 & 43. 51431 & 30024 \\
\hline & 7. 05431 & -184 & 6. 48334 & 2281 & 37.33120 & 26514 & 43.81455 & 28794 \\
\hline 5000 & 7.05247 & 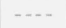 & 6.50615 & .... & 37.59634 & - & 44.10249 & 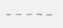 \\
\hline
\end{tabular}

TABLE 8. Ideal gas thermodynamic functions for $\mathrm{T}_{2} \mathrm{~S}$

\begin{tabular}{|l|l|l|l|l|}
\hline${ }^{\circ} \mathrm{K}$ & $\frac{C_{p}^{\circ}}{R}$ & $\frac{\left(H^{\circ}-E_{0}^{\circ}\right)}{R T}$ & $\frac{-\left(F^{\circ}-E_{0}^{\circ}\right)}{R T}$ & $\frac{S^{\circ}}{R}$ \\
\hline
\end{tabular}

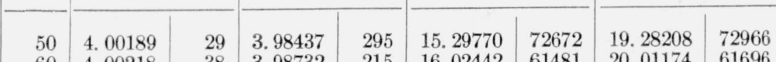
\begin{tabular}{l|l|l|l|l|l|l|l|l|}
60 & 4.00218 & 38 & 3.98732 & 215 & 16.02442 & 61481 & 20.01174 & 61696 \\
70 & 4.00256 & 66 & 3.98947 & 167 & 16.63923 & 53284 & 20,62870 & 53451
\end{tabular} \begin{tabular}{l|l|r|r|r|r|r|r|r|}
70 & 4.00256 & 66 & 3.98947 & 167 & 16.63923 & 53284 & 20,62870 & 53451 \\
80 & 4.00322 & 130 & 3.99114 & 141 & 17.17207 & 47017 & 21.16321 & 47158
\end{tabular} \begin{tabular}{l|l|l|l|l|l|l|l|l|}
90 & 4.00422 & 130 & 3.99114 & 141 & 17.17207 & 47017 & 21.16321 & 47158 \\
\hline
\end{tabular} \begin{tabular}{|l|l|l|l|l|l|l|l|l|}
100 & 4.00702 & 426 & 3.99386 & 137 & 18.06296 & 38072 & 22.05682 & 38210 \\
\hline
\end{tabular} \begin{tabular}{l|l|l|l|l|l|l|l|l|}
110 & 4.01128 & 657 & 3.99523 & 159 & 18.44368 & 34770 & 22.43892 & 34929 \\
\hline
\end{tabular} \begin{tabular}{l|l|l|l|l|l|l|l|l|}
110 & 4.01128 & 657 & 3.99523 & 159 & 18.44368 & 34770 & 22.43892 & 34029 \\
120 & 4.01785 & 923 & 3.99682 & 196 & 18.79138 & 31999 & 22.78821 & 32194 \\
\hline
\end{tabular} \begin{tabular}{l|l|r|l|l|l|l|l|l|}
130 & 4.02708 & 1205 & 3.99878 & 243 & 19.11137 & 29643 & 23.11015 & 29887 \\
\hline
\end{tabular} \begin{tabular}{|l|l|l|l|l|l|l|l|l|l|}
140 & 4.03913 & 1488 & 4.00121 & 301 & 19.40780 & 27615 & 23.40902 & 27916 \\
\hline
\end{tabular} \begin{tabular}{l|l|l|l|l|l|l|l|l|}
150 & 4.05401 & 1755 & 4.00422 & 365 & 19.68395 & 25854 & 23.68818 & 26218 \\
160 & 4.07156 & 1998 & 4.00787 & 432 & 19.94249 & 24310 & 23.95036 & 24743 \\
\hline
\end{tabular} \begin{tabular}{l|l|l|l|l|l|l|l|l}
160 & 4.07156 & 1998 & 4.00787 & 432 & 19.94249 & 24310 & 23.95036 & 24743 \\
170 & 4.09154 & 2213 & 4.01219 & 502 & 20.18559 & 22947 & 24.19779 & 23448 \\
\hline
\end{tabular} \begin{tabular}{l|l|l|l|l|l|l|l|l}
170 & 4.09154 & 2213 & 4.01219 & 502 & 20.18559 & 22947 & 24.19779 & 23448 \\
180 & 4.11367 & 2397 & 4.01721 & 570 & 20.41506 & 21735 & 24.43227 & 22305 \\
\hline
\end{tabular} \begin{tabular}{l|l|l|l|l|l|l|l|l}
180 & 4.11367 & 2397 & 4.01721 & 570 & 20.41506 & 21735 & 24.43227 & 22305 \\
190 & 4.13764 & 2555 & 4.02291 & 637 & 20.63241 & 20651 & 24.65532 & 21288
\end{tabular} \begin{tabular}{|l|l|l|l|l|l|l|l|l|}
200 & 4.16319 & 2687 & 4.02928 & 701 & 20.83892 & 19676 & 24.86820 & 20377 \\
\hline
\end{tabular} \begin{tabular}{l|l|l|l|l|l|l|l|l|}
210 & 4.19006 & 2798 & 4.03629 & 762 & 21.03568 & 18794 & 25.07197 & 19556 \\
\hline
\end{tabular} \begin{tabular}{|l|l|l|l|l|l|l|l|l|}
220 & 4.21804 & 2894 & 4.04391 & 820 & 21.22362 & 17994 & 25.26753 & 18814 \\
\hline
\end{tabular} \begin{tabular}{l|l|l|l|l|l|l|l|l}
220 & 4.21804 & 2894 & 4.04391 & 820 & 21.22362 & 17994 & 25.26753 & 18814 \\
230 & 4.24698 & 2975 & 4.05211 & 873 & 21.40356 & 17263 & 25.45567 & 18137
\end{tabular} \begin{tabular}{l|l|l|l|l|l|l|l|l|}
230 & 4.24698 & 2975 & 4.05211 & 873 & 21.40356 & 17263 & 25.45567 & 18137 \\
240 & 4.27673 & 3046 & 4.06084 & 925 & 21.57619 & 16596 & 25.63704 & 17520
\end{tabular} \begin{tabular}{|l|l|l|l|r|r|r|r|r|}
250 & 4.30719 & 3109 & 4.07009 & 971 & 21.74215 & 15982 & 25.81224 & 16954 \\
\hline
\end{tabular} \begin{tabular}{|l|l|l|l|l|l|l|l|l|}
260 & 4.33828 & 3167 & 4.07980 & 1016 & 21.90197 & 15416 & 25.98178 & 16432 \\
\hline
\end{tabular} \begin{tabular}{l|l|l|l|l|l|l|l|l|}
260 & 4.33828 & 3167 & 4.07980 & 1016 & 21.90197 & 15416 & 25.98178 & 15452 \\
270 & 4.36995 & 3220 & 4.08996 & 1057 & 22.05613 & 14894 & 26.14610 & 15950 \\
\hline
\end{tabular} \begin{tabular}{l|l|l|l|l|l|l|l|l|}
280 & 4.30215 & 3269 & 4.10053 & 1097 & 22.20507 & 14408 & 26.30560 & 15505 \\
\hline
\end{tabular} \begin{tabular}{l|l|l|l|l|l|l|l|l|}
280 & 4.40215 & 3269 & 4.10053 & 1097 & 22.20507 & 14408 & 26.30560 & 15505 \\
290 & 4.43484 & 3315 & 4.11150 & 1132 & 22.34915 & 13958 & 26.46065 & 15091
\end{tabular} \begin{tabular}{|l|l|l|l|l|l|l|l|l|}
300 & 4.46799 & 3358 & 4.12282 & 1168 & 22.48873 & 13537 & 26.61156 & 14705 \\
\hline
\end{tabular} \begin{tabular}{|l|l|l|l|l|l|l|l|l|}
310 & 4.50157 & 3397 & 4.13450 & 1200 & 22.62410 & 13146 & 26.75861 & 14345 \\
\hline
\end{tabular} \begin{tabular}{l|l|l|l|l|l|l|l|l|}
310 & 4.50157 & 3397 & 4.13450 & 1200 & 22.62410 & 13146 & 26.75861 & 14345 \\
320 & 4.53554 & 3433 & 4.14650 & 1231 & 22.75556 & 12778 & 26.90206 & 14009 \\
\hline
\end{tabular} \begin{tabular}{l|l|l|l|l|l|l|l|l|}
320 & 4.53554 & 3433 & 4.14650 & 1231 & 22.75556 & 1277 & 26.90206 & 14009 \\
330 & 4.56987 & 3466 & 4.15881 & 1260 & 22.88334 & 12434 & 27.04215 & 13694
\end{tabular} \begin{tabular}{l|l|l|l|l|l|l|l|l}
330 & 4.56987 & 3466 & 4.15881 & 1260 & 22.88334 & 12434 & 27.04215 & 13694 \\
340 & 4.60453 & 3494 & 4.17141 & 1287 & 23.00768 & 12110 & 27.17909 & 13398
\end{tabular} \begin{tabular}{|l|l|l|l|l|l|l|l|l|}
350 & 4.63947 & 3518 & 4.18428 & 1313 & 23.12878 & 11806 & 27.31307 & 13119 \\
\hline
\end{tabular} \begin{tabular}{|l|l|l|l|l|l|l|l|l|}
360 & 4.67465 & 3539 & 4.19741 & 1338 & 23.24684 & 11519 & 27.44426 & 12856 \\
\hline
\end{tabular} \begin{tabular}{l|l|l|l|l|l|l|l|l|}
360 & 4.67465 & 3539 & 4.19741 & 1338 & 23.24684 & 11519 & 27.44426 & 12856 \\
370 & 4.71004 & 3554 & 4.21079 & 1360 & 23.36203 & 11247 & 27.57282 & 12608 \\
\hline
\end{tabular} \begin{tabular}{|l|l|l|l|l|l|l|l|l|}
370 & 4.71004 & 3554 & 4.21079 & 1360 & 23.36203 & 11247 & 27.57282 & 12608 \\
\hline 380 & 4.74558 & 3565 & 4.22439 & 1383 & 23.47450 & 10991 & 27.69890 & 12373
\end{tabular} \begin{tabular}{l|l|l|l|l|l|l|l|l|}
380 & 4.78123 & 3571 & 4.23822 & 1402 & 23.58441 & 10748 & 27.82263 & 12150
\end{tabular} \begin{tabular}{|l|l|l|l|l|l|l|l|l|}
4400 & 4.81694 & 17793 & 4.25224 & 7265 & 23.69189 & 50500 & 27.94413 & 57765 \\
\hline
\end{tabular} \begin{tabular}{|l|l|l|l|l|l|l|l|l|}
\hline 450 & 4.99487 & 17314 & 4.32489 & 7571 & 24.19689 & 45957 & 28.52178 & 53529 \\
\hline
\end{tabular} \begin{tabular}{l|l|l|l|l|l|l|l|l|}
500 & 5.16801 & 16442 & 4.40060 & 7732 & 24.65646 & 42304 & 29.05707 & 50035 \\
\hline
\end{tabular} \begin{tabular}{l|l|l|l|l|l|l|l|l|}
550 & 5.33243 & 15327 & 4.47792 & 7768 & 25.07950 & 39296 & 29.55742 & 47064 \\
600 & 5.48570 & 14102 & 4.55560 & 7705 & 25.47246 & 36770 & 30.02806 & 44475
\end{tabular} \begin{tabular}{l|l|l|l|l|l|l|l|l|}
650 & 5.62672 & 12857 & 4.63265 & 7567 & 25.84016 & 34609 & 30.47281 & 42176 \\
\hline
\end{tabular} \begin{tabular}{|l|l|l|l|l|l|l|l|l|}
700 & 5.75529 & 11658 & 4.70832 & 7375 & 26.18625 & 32737 & 30.89457 & 40112 \\
\hline
\end{tabular} \begin{tabular}{l|l|l|l|l|l|l|l|l|l|}
750 & 5.87187 & 10533 & 4.78207 & 7146 & 26.51362 & 31092 & 31.29569 & 38238 \\
\hline
\end{tabular} \begin{tabular}{l|l|l|l|l|l|l|l|l|}
800 & 5.97720 & 9505 & 4.85353 & 6894 & 26.82454 & 29633 & 31.67807 & 36527 \\
850 & 6.07225 & 8573 & 4.92247 & 6630 & 27.12087 & 28325 & 32.04334 & 34955
\end{tabular} \begin{tabular}{|l|l|l|l|l|l|l|l|l|}
900 & 6.15798 & 7738 & 4.98877 & 6361 & 27.40412 & 27144 & 32.39289 & 33505 \\
\hline
\end{tabular} \begin{tabular}{l|l|l|l|l|l|l|l|l|}
950 & 6.23536 & 6996 & 5.05238 & 6092 & 27.67556 & 26072 & 32.72794 & 32164 \\
\hline
\end{tabular} \begin{tabular}{|l|l|l|l|l|l|l|l|l|}
1000 & 6.30532 & 6334 & 5.11330 & 5830 & 27.93628 & 25090 & 33.04958 & 30920
\end{tabular} \begin{tabular}{|l|l|l|l|l|l|l|l|l|l|l|}
\hline 1050 & 6.36866 & 5749 & 5.17160 & 5574 & 28.18718 & 24188 & 33.35878 & 29762 \\
\hline
\end{tabular} \begin{tabular}{l|l|l|l|l|l|l|l|l|l|}
11100 & 6.42615 & 5230 & 5.22734 & 5327 & 28.42906 & 23354 & 33.65640 & 28682
\end{tabular} \begin{tabular}{|l|l|l|l|l|l|l|l|l|}
\hline 1150 & 6.47845 & 4771 & 5.28061 & 5092 & 28.66260 & 22583 & 33.94322 & 27675 \\
\hline
\end{tabular} \begin{tabular}{|l|l|l|l|l|l|l|l|l|}
\hline 1200 & 6.52616 & 8363 & 5.33153 & 9521 & 28.88843 & 43057 & 34.21997 & 52577 \\
\hline
\end{tabular} \begin{tabular}{|l|l|l|l|l|l|l|l|l|l|}
1300 & 6.60979 & 7069 & 5.42674 & 8709 & 29.31900 & 40540 & 34.74574 & 49249 \\
\end{tabular} \begin{tabular}{|l|l|l|l|l|l|l|l|l|}
1400 & 6.68048 & 6040 & 5.51383 & 7984 & 29.72440 & 38317 & 35.23823 & 46302 \\
\hline
\end{tabular} \begin{tabular}{|l|l|l|l|l|l|l|l|l|}
1500 & 6.74088 & 5214 & 5.59367 & 7337 & 30.10757 & 36339 & 35.70125 & 43675 \\
\hline
\end{tabular} \begin{tabular}{|l|l|l|l|l|l|l|l|l|l|}
1000 & 6.79302 & 4546 & 5.66704 & 6760 & 30.47096 & 34561 & 36.13800 & 41322
\end{tabular} \begin{tabular}{|l|l|l|l|l|l|l|l|l|}
\hline 1700 & 6.83848 & 4001 & 5.73464 & 6246 & 30.81657 & 32958 & 36.55122 & 39203 \\
\hline
\end{tabular} \begin{tabular}{|l|l|l|l|l|l|l|l|l|l|}
1800 & 6.87849 & 3552 & 5.79710 & 5787 & 31.14615 & 31500 & 36.94325 & 37288 \\
\hline
\end{tabular} \begin{tabular}{l|l|l|l|l|l|l|l|l|l|}
1900 & 6.91401 & 3181 & 5.85497 & 5376 & 31.46115 & 30171 & 37.31613 & 35546 \\
\hline
\end{tabular} \begin{tabular}{|l|l|l|l|l|l|l|l|l|l|}
2000 & 6. 94582 & 2868 & 5.90873 & 5008 & 31.70286 & 28951 & 37.67159 & 33960 \\
\hline
\end{tabular} \begin{tabular}{|l|l|l|l|l|l|l|l|l|}
2200 & 7.00056 & 2384 & 6.00558 & 4378 & 32.33067 & 26793 & 38.33625 & 31172 \\
\hline
\end{tabular} \begin{tabular}{|l|l|l|l|l|l|l|l|l|}
22300 & 7.02440 & 2192 & 6.04936 & 4109 & 32.59860 & 25834 & 38.64797 & 29943 \\
\hline
\end{tabular} \begin{tabular}{l|l|l|l|l|l|l|l|l|}
2400 & 7.04632 & 2029 & 6.09045 & 3865 & 32.85694 & 24941 & 38.94740 & 28806 \\
\hline
\end{tabular} \begin{tabular}{l|l|l|l|l|l|l|l|l}
2500 & 7.06661 & 1886 & 6.12910 & 3642 & 33.10635 & 24111 & 39.23546 & 27753 \\
\end{tabular} \begin{tabular}{|l|l|l|l|l|l|l|l|l|}
2600 & 7.08547 & 1763 & 6.16552 & 3441 & 33.34746 & 23334 & 39.51299 & 26774 \\
\hline
\end{tabular} \begin{tabular}{|l|l|l|l|l|l|l|l|l|}
2700 & 7.10310 & 1654 & 6.19993 & 3255 & 33.58080 & 22607 & 39.78073 & 25863 \\
2800 & 7.11964 & 1559 & 6.23248 & 3086 & 33.80687 & 21925 & 40.03936 & 25011 \\
\hline
\end{tabular} \begin{tabular}{|l|l|l|l|l|l|l|l|l|}
2900 & 7.13523 & 1473 & 6.26334 & 2931 & 34.02612 & 21283 & 40.28947 & 24214 \\
\hline
\end{tabular} \begin{tabular}{|l|l|l|l|l|l|l|l|l|}
3000 & 7.14996 & 2729 & 6.29265 & 5445 & 34.23895 & 40789 & 40.53161 & 46234
\end{tabular}

\begin{tabular}{|l|l|l|l|l|l|l|l|l|}
\hline 3200 & 7.17725 & 2484 & 6.34710 & 4958 & 34.64684 & 38630 & 40.99395 & 43587 \\
\hline
\end{tabular} \begin{tabular}{|l|l|l|l|l|l|l|l|l|}
3400 & 7.20209 & 2283 & 6.39668 & 4538 & 35.03314 & 36693 & 41.42982 & 41232 \\
\hline
\end{tabular} \begin{tabular}{|l|l|l|l|l|l|l|l|l|l|}
3600 & 7.22492 & 2115 & 6.44206 & 4177 & 35.40007 & 34944 & 41.84214 & 39120 \\
\hline
\end{tabular} \begin{tabular}{|l|l|l|l|l|l|l|l|l|l|}
38000 & 7.260080 & 1849 & 6.52244 & 3584 & 36.08308 & 31911 & 42.60552 & 35496 \\
\hline
\end{tabular} \begin{tabular}{|l|l|l|l|l|l|l|l|l|}
\hline 4200 & 7.28429 & 1741 & 6.55828 & 3340 & 36.40219 & 30587 & 42.96048 & 33927 \\
\hline
\end{tabular} \begin{tabular}{|l|l|l|l|l|l|l|l|l|}
4400 & 7.30170 & 1645 & 6.59168 & 3123 & 36.70806 & 29371 & 43.29975 & 32494 \\
\hline
\end{tabular} \begin{tabular}{|l|l|l|l|l|l|l|l|l|l|}
4600 & 7.31815 & 1558 & 6.62291 & 2930 & 37.00177 & 28249 & 43.62469 & 31179
\end{tabular} \begin{tabular}{|l|l|l|l|l|l|l|l|l|}
4800 & 7.33373 & 1480 & 6.65221 & 2756 & 37.28426 & 27212 & 43.93648 & 29968 \\
\hline
\end{tabular} 


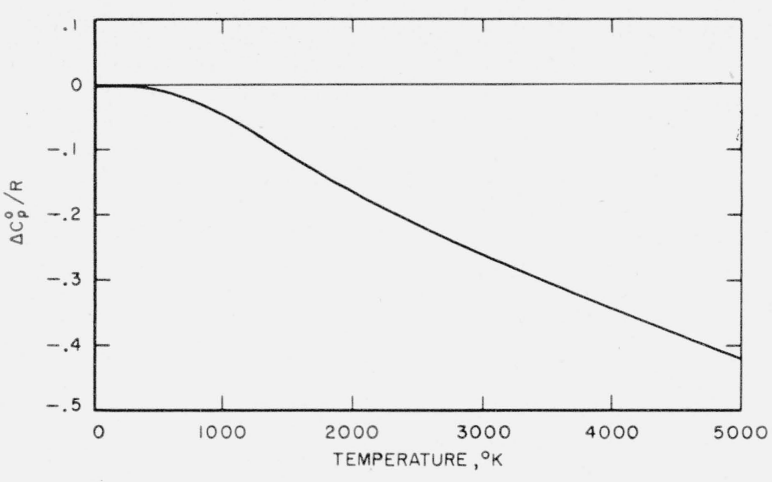

FIgure 1. Comparison of ideal gas heat capacity functions for $\mathrm{H}_{2} \mathrm{~S}$.

The SEAC values lie on the base line. The curve represents the difference between the rigid-rotator, harmonic-oscillator calculations, and the SEAC between the
calculations.

The uncertainty in the calculated anharmonicity and rotation-vibration coupling constants for HDS, H'TS, and DTS depends on the validity of using HDO as a reference molecule in the isotope expressions for $X_{i j}$ and $\delta_{i}$. The uncertainty in these constants for $\mathrm{T}_{2} \mathrm{~S}$, estimated from the differences between experimental and calculated values for analogous molecules, is probably below 20 percent. The resulting uncertainty in the fundamentals for $\mathrm{T}_{2} \mathrm{~S}$ are approximately 1 percent. assuming negligible uncertainty in the calculated zero order frequencies. At temperatures below $1,000^{\circ} \mathrm{K}$ this results in an uncertainty in the third place to the right of the decimal for the tabulated thermodynamic functions of $\mathrm{T}_{2} \mathrm{~S}$. The uncertainty in the tabulated results for $\mathrm{H}_{2} \mathrm{~S}$ and $\mathrm{D}_{2} \mathrm{~S}$ would not exceed that for $\mathrm{T}_{2} \mathrm{~S}$. At higher temperatures the uncertainty in the thermodynamic functions is larger, due to neglected higher order terms in the energy level expressions, and the omission of the effect of rotational cut-off.

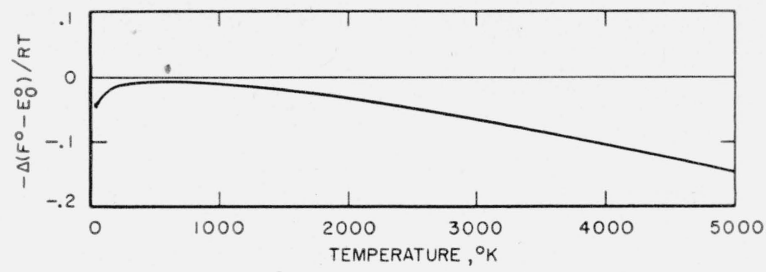

Figure 2. Comparison of ideal gas free energy functions for $\mathrm{H}_{2} \mathrm{~S}$.

The SEAC values lie on the base line. The curve represents the difference between the rigid-rotator, harmonic-oscillator calculations, and the SEAC calculations.

Two sets of calculations of thermodynamic functions were made on the SEAC: one set for only harmonic oscillator, rigid rotator contributions; and the tabulated set, which includes these plus first order effects for centrifugal stretching, vibrational anharmonicity, rotation-vibration coupling and low temperature nonclassical rotation terms. A comparison of the two different sets of calculations of $C_{p}^{\circ} / R$ and $-\left(F^{\circ}-E_{0}^{\circ}\right) / R T$ for $\mathrm{H}_{2} \mathrm{~S}$ is given in figures 1 and 2 from $50^{\circ}$ to $5,000^{\circ} \mathrm{K}$.

\section{References}

[1] H. C. Allen (private communication).

[2] A. S. Friedman and L. Haar, J. Chem. Phys. 22, 2051 (1954).

[3] L. Haar and A. S. Friedman, J. Chem. Phys. 23, 869 (1955).

[4] K. F. Stripp and J. G. Kirkwood, J. Chem. Phys. 19, 1131 (1951).

[5] H. W. Woolley, Dissertation, Univ. of Michigan, 1955.

[6] E. Wichers, J. Am. Chem. Soc. 74, 2447 (1952).

[7] J. Mattauch and S. Fluegge, Nuclear physics tables and an introduction to nuclear physics (Interscience Publishers, Inc., New York, N. Y., 1946).

Washington, July 14, 1955. 